Теорія Ймовір. та Матем. Статист. Вип. 71, 2004
Theor. Probability and Math. Statist.

No. 71, 2005, Pages 63-79

S 0094-9000(05)00648-4

Article electronically published on December 28, 2005

\title{
MOTIONS WITH FINITE VELOCITY ANALYZED WITH ORDER STATISTICS AND DIFFERENTIAL EQUATIONS
}

UDC 519.21

\author{
A. DE GREGORIO, E. ORSINGHER, AND L. SAKHNO
}

\begin{abstract}
The aim of this paper is to derive the explicit distribution of the position of randomly moving particles on the line and in the plane (with different velocities taken cyclically) by means of order statistics and by studying suitable problems of differential equations. The two approaches are compared when both are applicable (case of the telegraph process). In some specific cases (alternating motions with skipping) it is possible to use the order statistics approach also to solve the equations governing the distribution. Finally, the approach based on order statistics is also applied in order to obtain the distribution of the position in the case of planar motion with three velocities conditioned on the number of changes of directions recorded.
\end{abstract}

\section{INTRODUCTION}

The analysis of random motions with finite velocity can be performed either by resolving initial-value problems for the equation governing the probability distribution or by exploiting the order statistics.

The prototype of processes related to this type of motions is the telegraph process $T=T(t), t>0$, introduced by Goldstein in 2 and examined over the years by different authors (see, for example, [1]). The approach used in studying the

$$
\mathrm{P}\{T(t) \in d x\}=p(x, t) d x
$$

is based on the solution to the following Cauchy problem:

$$
\left\{\begin{array}{l}
\frac{\partial^{2} p}{\partial t^{2}}+2 \lambda \frac{\partial p}{\partial t}=c^{2} \frac{\partial^{2} p}{\partial x^{2}} \\
p(x, 0)=\delta(x), \\
p_{t}(x, 0)=0 .
\end{array}\right.
$$

In this special case it is easy to obtain $p=p(x, t)$ also by means of order statistics. This approach permits us to derive also

$$
p_{k}(x, t) d x=\mathrm{P}\{T(t) \in d x, N(t)=k\},
$$

where $N(t)$ is the number of changes of direction of motion occurring up to time $t$.

We will show below that the joint distributions (1.2) can also be calculated analytically by establishing that the function

$$
G(u, x, t)=\sum_{k=0}^{\infty} u^{k} p_{k}(x, t)
$$

2000 Mathematics Subject Classification. Primary 60K99; Secondary 62G30, 35L25.

Key words and phrases. Order statistics, Bessel functions of higher order, random motions.

This work was partially supported by the NATO grant PST.CLG.976361. 
is a solution to

$$
\left\{\begin{array}{l}
\frac{\partial^{2} G}{\partial t^{2}}+2 \lambda \frac{\partial G}{\partial t}=c^{2} u^{2} \frac{\partial^{2} G}{\partial x^{2}}, \\
G(u, x, 0)=\delta(x), \\
G_{t}(u, x, 0)=0 .
\end{array}\right.
$$

In some cases the approach based on order statistics seems to be the only possible instrument for obtaining the distribution

$$
p(x, t) d x=\mathrm{P}\{X(t) \in d x\}
$$

where $X(t), t>0$, is one-dimensional random motion with finite velocities. If the possible velocities are $c_{k}, k=1,2, \ldots, n$, and the changes of motion are paced by a Poisson process, then the distributions

$$
\begin{gathered}
p_{k}^{j}(x, t) d x=\mathrm{P}\left\{X(t) \in d x, N(t)=k, V(t)=c_{j}\right\}, \\
k \geq 0, \quad j=1,2, \ldots, n,
\end{gathered}
$$

with $V(t)$ being the current velocity, can be explicitly obtained (at least for a small number $n$ of possible velocities) for any $j$ by means of order statistics.

In the case where $n=4, c_{1}=c, c_{2}=0, c_{3}=-c$, and $c_{4}=0$ this has been done in [4]. This type of motion (alternating with randomly distributed stops) emerges in the analysis of cyclic planar motions with orthogonal directions because a particle while moving horizontally does not change its vertical coordinate. We here consider the case where $n=4, c_{1}=c_{2}=c$, and $c_{3}=c_{4}=-c$, which resembles somehow the telegraph process. This is a four-state motion, symmetrically distributed around the starting point. Its probability distribution $p$ is a solution to the fourth-order equation:

$$
\left[\left(\frac{\partial}{\partial t}+\lambda\right)^{2}-c^{2} \frac{\partial^{2}}{\partial x^{2}}-\lambda^{2}\right]\left[\left(\frac{\partial}{\partial t}+\lambda\right)^{2}-c^{2} \frac{\partial^{2}}{\partial x^{2}}+\lambda^{2}\right] p=0 .
$$

Equation (1.7) must be compared with

$$
\left(\frac{\partial}{\partial t}+\lambda\right)^{2}\left[\left(\frac{\partial}{\partial t}+\lambda\right)^{2}-c^{2} \frac{\partial^{2}}{\partial x^{2}}\right] p-\lambda^{4} p=0
$$

which is satisfied by the law of process with two steps interrupted by stops.

It is remarkable that for a process with $n-2$ stops, one step forward and one step backward, the equation satisfied by $p$ reads

$$
\left[\left(\frac{\partial}{\partial t}+\lambda\right)^{n}-\lambda^{n}-c^{2}\left(\frac{\partial}{\partial t}+\lambda\right)^{n-2} \frac{\partial^{2}}{\partial x^{2}}\right] p=0
$$

and can be reduced, by means of the exponential transformation $w=e^{-\lambda t} p$, to the simple form:

$$
\frac{\partial^{n-2}}{\partial t^{n-2}}\left[\frac{\partial^{2}}{\partial t^{2}}-c^{2} \frac{\partial^{2}}{\partial x^{2}}\right] p-\lambda^{n} p=0 .
$$

The reader can ascertain that for $n=2,(1.9)$ coincides with the telegraph equation (1.1).

We have obtained, at least in the one-dimensional cases mentioned above, explicit distributions. Let us list the explicit expressions for the absolutely continuous components of the distribution of the process (with stops) which have been derived so far. We denote by

$$
p_{l}(x, t)=p_{l}
$$

the density of the process with $l$ states $(l-2$ stops $)$. Therefore $p_{2}=p_{2}(x, t)$ is the law of the telegraph process, $p_{3}(x, t)$ is a three-state motion with one stop and $p_{4}(x, t)$ is related 
to the case of forward and backward displacements interrupted by stops. We have the following formulas:

$$
\begin{aligned}
& p_{2}(x, t)=e^{-\lambda t} \sum_{k=1}^{\infty}\left(\frac{\lambda t}{2 k}+1\right)\left(\frac{\lambda}{2 c}\right)^{2 k-1} \frac{\left(c^{2} t^{2}-x^{2}\right)^{k-1}}{(k-1) !^{2}}, \quad|x|<c t, \\
& p_{3}(x, t)=e^{-\lambda t} \sum_{k=1}^{\infty}\left(\frac{\lambda t}{3 k}+1+\frac{1}{\lambda} \frac{\partial}{\partial t}\right)\left(\frac{\lambda}{c}\right)^{3 k-1} \frac{1}{2^{2 k-1}(k-1) !^{3}} \\
& \times \int_{\max (-x, x)}^{c t}(c t-w)^{k-1}\left(w^{2}-x^{2}\right)^{k-1} d w, \quad|x|<c t, \\
& p_{4}(x, t)=e^{-\lambda t} \sum_{k=1}^{\infty}\left(\frac{\lambda t}{4 k}+1+\frac{1}{\lambda} \frac{\partial}{\partial t}+\frac{1}{\lambda^{2}} \frac{\partial^{2}}{\partial t^{2}}\right)\left(\frac{\lambda}{c}\right)^{4 k-1} \\
& \times \frac{1}{2^{2 k-1}(k-1) !^{2}(2 k-1) !} \\
& \times \int_{\max (-x, x)}^{c t}(c t-w)^{2 k-1}\left(w^{2}-x^{2}\right)^{k-1} d w, \quad|x|<c t .
\end{aligned}
$$

Formula (1.11a) has been obtained by many different authors and by different methods and is usually represented in terms of Bessel functions as follows:

$$
p_{2}(x, t)=\frac{e^{-\lambda t}}{2 c}\left[\lambda I_{0}\left(\frac{\lambda}{c} \sqrt{c^{2} t^{2}-x^{2}}\right)+\frac{\partial}{\partial t} I_{0}\left(\frac{\lambda}{c} \sqrt{c^{2} t^{2}-x^{2}}\right)\right],
$$

Result (1.11c) appears explicitly in [4]. In this paper we obtain the distribution $\hat{p}_{4}=\hat{p}_{4}(x, t)$ for the process with velocities $c_{1}=c_{2}=c$ and $c_{3}=c_{4}=-c$ and this reads:

$$
\begin{gathered}
\hat{p}_{4}(x, t)=e^{-\lambda t} \sum_{k=1}^{\infty}\left(\frac{\lambda t}{4 k}+1+\frac{1}{\lambda} \frac{\partial}{\partial t}+\frac{1}{\lambda^{2}} \frac{\partial^{2}}{\partial t^{2}}\right)\left(\frac{\lambda}{2 c}\right)^{4 k-1} \frac{\left(c^{2} t^{2}-x^{2}\right)^{2 k-1}}{(2 k-1) !^{2}}, \\
|x|<c t
\end{gathered}
$$

The last section of the paper is devoted to the analysis of the joint distributions

$$
\begin{gathered}
p_{3 n-j}(x, y, t) d x d y=\mathrm{P}\{X(t) \in d x, Y(t) \in d y \mid N(t)=3 n-j\} \\
\text { for } n \geq 1, j=1,2, \quad \text { and } n \geq 2, j=3,
\end{gathered}
$$

of a planar cyclic motion with three directions. The conditional distributions have been obtained by means of order statistics and from them the unconditional distribution, expressed in terms of Bessel functions of order three, has been derived.

\section{The TElegraph PROCESS AND THE APPROACH BASED ON ORDER STATISTICS}

The telegraph process is defined by

$$
T(t)=V(0) \int_{0}^{t}(-1)^{N(s)} d s,
$$

where $V(0)$ is a two-valued r.v. (with values $\pm c$ taken with probability $1 / 2$ ) and $N(t)$, $t>0$, is the number of events of a Poisson process in $[0, t]$. The r.v.'s $N(t)$ and $V(0)$ are independent, and we can explicitly obtain the distributions

$$
p_{k}(x, t) d x=\mathrm{P}\{T(t) \in d x, N(t)=k\}, \quad k \geq 0,
$$

with $x \in(-c t, c t)$, in two different ways. 
Our first approach is analytical and profits from the fact that the distribution of (2.1) is well known and reads:

$$
\begin{aligned}
p(x, t)= & \frac{e^{-\lambda t}}{2 c}\left[\lambda I_{0}\left(\frac{\lambda}{c} \sqrt{c^{2} t^{2}-x^{2}}\right)+\frac{\partial}{\partial t} I_{0}\left(\frac{\lambda}{c} \sqrt{c^{2} t^{2}-x^{2}}\right)\right] I_{\{|x|<c t\}} \\
& +\frac{e^{-\lambda t}}{2}[\delta(x-c t)+\delta(x+c t)],
\end{aligned}
$$

where $I_{0}$ is the zero-order modified Bessel function.

We introduce the distributions

$$
\left\{\begin{array}{l}
f_{k}(x, t) d x=\mathrm{P}\{T(t) \in d x, V(t)=c, N(t)=k\}, \\
b_{k}(x, t) d x=\mathrm{P}\{T(t) \in d x, V(t)=-c, N(t)=k\},
\end{array}\right.
$$

where $k \geq 1$ and

$$
V(t)=V(0)(-1)^{N(t)}, \quad t>0
$$

represents the current velocity of the particle performing the motion (2.1).

The distributions (2.4) are solutions to the following difference-differential system:

$$
\left\{\begin{array}{l}
\frac{\partial f_{k}}{\partial t}=-c \frac{\partial f_{k}}{\partial x}+\lambda\left(b_{k-1}-f_{k}\right) \\
\frac{\partial b_{k}}{\partial t}=c \frac{\partial b_{k}}{\partial x}+\lambda\left(f_{k-1}-b_{k}\right) .
\end{array}\right.
$$

In terms of the function $w_{k}=f_{k}-b_{k}$ and $p_{k}=f_{k}+b_{k}$ the system (2.6) can be rewritten in the following manner:

$$
\left\{\begin{array}{l}
\frac{\partial p_{k}}{\partial t}=-c \frac{\partial w_{k}}{\partial x}+\lambda p_{k-1}-\lambda p_{k} \\
\frac{\partial w_{k}}{\partial t}=-c \frac{\partial p_{k}}{\partial x}-\lambda w_{k-1}-\lambda w_{k}
\end{array}\right.
$$

From (2.7), by successive derivations, we obtain the following second-order partial difference-differential equation:

$$
\frac{\partial^{2} p_{k}}{\partial t^{2}}=c^{2} \frac{\partial^{2} p_{k}}{\partial x^{2}}-2 \lambda \frac{\partial p_{k}}{\partial t}-\lambda^{2} p_{k}+\lambda^{2} p_{k-2}, \quad k \geq 0
$$

with $p_{-1}=p_{-2}=0$.

An alternative form of equation (2.8) is

$$
\left(\frac{\partial}{\partial t}+\lambda\right)^{2} p_{k}=c^{2} \frac{\partial^{2} p_{k}}{\partial x^{2}}+\lambda^{2} p_{k-2}
$$

and can easily be reduced, by means of the exponential transformation $p_{k}=e^{-\lambda t} w_{k}$ to the following one:

$$
\frac{\partial^{2} w_{k}}{\partial t^{2}}=c^{2} \frac{\partial^{2} w_{k}}{\partial x^{2}}+\lambda^{2} w_{k-2}
$$

Now we have our first theorem.

Theorem 2.1. The generating function

$$
G(u, x, t)=\sum_{k=0}^{\infty} u^{k} p_{k}(x, t)
$$


is the solution to the initial value problem:

$$
\left\{\begin{array}{l}
\left(\frac{\partial}{\partial t}+\lambda\right)^{2} G=c^{2} \frac{\partial^{2} G}{\partial x^{2}}+\lambda^{2} u^{2} G \\
G(u, x, 0)=\delta(x) \\
\left.\frac{\partial}{\partial t} G(u, x, t)\right|_{t=0}=0
\end{array}\right.
$$

Proof. It suffices to multiply both terms of (2.9) by $u^{k}$ and sum up with respect to $k$. From (2.11) it is clear that $G$ satisfies the initial conditions in $(2.12)$ because $p_{0}(x, 0)=$ $\delta(x)$ and $p_{k}(x, 0)=0, k \geq 1$.

Corollary 2.1. By performing the same steps as in the derivation of (2.3) it is immediate that the generating function $G$ has the form

$$
\begin{aligned}
G(u, x, t)= & \frac{e^{-\lambda t}}{2 c}\left[\lambda u I_{0}\left(\frac{\lambda u}{c} \sqrt{c^{2} t^{2}-x^{2}}\right)+\frac{\partial}{\partial t} I_{0}\left(\frac{\lambda u}{c} \sqrt{c^{2} t^{2}-x^{2}}\right)\right] I_{\{|x|<c t\}} \\
& +\frac{e^{-\lambda t}}{2}[\delta(x-c t)+\delta(x+c t)] .
\end{aligned}
$$

By expanding both members of (2.13) we obtain

$$
\begin{aligned}
G(u, x, t)= & \sum_{k=0}^{\infty} u^{k} p_{k}(x, t) \\
= & \frac{e^{-\lambda t}}{2 c}\left[\lambda u \sum_{k=0}^{\infty}\left(\frac{\lambda u}{2 c}\right)^{2 k} \frac{1}{k !^{2}}\left(c^{2} t^{2}-x^{2}\right)^{k}\right. \\
& \left.+\sum_{k=1}^{\infty}\left(\frac{\lambda u}{2 c}\right)^{2 k} \frac{1}{k !^{2}} \frac{\partial}{\partial t}\left(c^{2} t^{2}-x^{2}\right)^{k}\right] \\
& +\frac{e^{-\lambda t}}{2}[\delta(x-c t)+\delta(x+c t)] .
\end{aligned}
$$

The second sum in (2.14) begins with $k=1$ because the derivation causes the term $(-1)$ ! to appear.

Corollary 2.2. From (2.14) we extract the following explicit formulas:

$$
\begin{cases}p_{2 k+1}(x, t)=e^{-\lambda t}\left(\frac{\lambda}{2 c}\right)^{2 k+1} \frac{1}{k !^{2}}\left(c^{2} t^{2}-x^{2}\right)^{k}, & |x|<c t, k \geq 0 \\ p_{2 k}(x, t)=e^{-\lambda t} \frac{\lambda^{2 k}}{(2 c)^{2 k+1}} \frac{1}{k !^{2}} \frac{\partial}{\partial t}\left(c^{2} t^{2}-x^{2}\right)^{k}, & |x|<c t, k \geq 1 .\end{cases}
$$

It can be checked that

$$
\int_{-c t}^{+c t} p_{2 k+j}(x, t) d x=\mathrm{P}\{N(t)=2 k+j\}, \quad j=0,1, \ldots
$$

From (2.15) we also have that

$$
\begin{gathered}
\mathrm{P}\{T(t) \in d x \mid N(t)=2 k+1\}=d x \frac{(2 k+1) !}{k !^{2}} \frac{\left(c^{2} t^{2}-x^{2}\right)^{k}}{(2 c t)^{2 k+1}}, \\
|x|<c t, \quad k \geq 0,
\end{gathered}
$$


and

$$
\begin{gathered}
\mathrm{P}\{T(t) \in d x \mid N(t)=2 k\}=d x \frac{(2 k) !}{k !(k-1) !} \frac{c t\left(c^{2} t^{2}-x^{2}\right)^{k-1}}{(2 c t)^{2 k}}, \\
|x|<c t, \quad k \geq 1 .
\end{gathered}
$$

The reader can also note a somewhat surprising fact that

$$
\mathrm{P}\{T(t) \in d x \mid N(t)=2 k+1\}=\mathrm{P}\{T(t) \in d x \mid N(t)=2 k+2\} .
$$

We now show that (2.15) can also be obtained in a completely different way by means of order statistics. Let $N(t)=n$ and consider the motion up to the time moment $t$. We first remark that the particle performing the process $T$ makes steps of the form

$$
\pm c t\left(T_{(j)}-T_{(j-1)}\right)
$$

where $t T_{(j)}$ is the time of the $j$-th Poisson event. $T_{(j)}, 1 \leq j \leq n$, can also be viewed as the $j$-th order statistics from the uniform law in $[0,1]$. Because of the exchangeability of the r.v.'s $T_{(j)}-T_{(j-1)}$ we can reorder the $n^{+}$forward steps and put them together (as well as the backward ones).

In this way the displacement, when $n$ changes of direction occur, is equivalent in distribution to

$$
T(t)=c t\left[T_{\left(n^{+}\right)}-\left(1-T_{\left(n^{+}\right)}\right)\right],
$$

where $T_{\left(n^{+}\right)}$is the $n^{+}$-th order statistic. From (2.20) we clearly have

$$
\begin{aligned}
& \mathrm{P}\{T(t)<x \mid N(t)=n, V(0)= \pm c\} \\
& \quad=\mathrm{P}\left\{T_{\left(n^{+}\right)}<\frac{1}{2}\left(1+\frac{x}{c t}\right) \mid N(t)=n, V(0)= \pm c\right\} .
\end{aligned}
$$

The distribution of the order statistic $T_{\left(n^{+}\right)}$has the density

$$
f_{T_{(n+)}}(w)=\frac{n !}{\left(n^{+}-1\right) !\left(n-n^{+}\right) !} w^{n^{+}-1}(1-w)^{n-n^{+}}, \quad 0<w<1,
$$

where the connection between $n$ and $n^{+}$depends on the sign of the initial velocity. By combining (2.21) and (2.22) we have

$$
\begin{aligned}
& \mathrm{P}\{T(t) \in d x \mid N(t)=n, V(0)= \pm c\} \\
& =d x \frac{n !}{(2 c t)\left(n^{+}-1\right) !\left(n-n^{+}\right) !}\left[\frac{1}{2}\left(1+\frac{x}{c t}\right)\right]^{n^{+}-1}\left[1-\frac{1}{2}\left(1+\frac{x}{c t}\right)\right]^{n-n^{+}} \\
& \quad=d x \frac{n !}{(2 c t)^{n}\left(n^{+}-1\right) !\left(n-n^{+}\right) !}(c t+x)^{n^{+}-1}(c t-x)^{n-n^{+}} .
\end{aligned}
$$

In particular, if $n=2 k+1$, and $V(0)=c, n^{+}=k+1$, then we obtain

$$
\begin{gathered}
\mathrm{P}\{T(t) \in d x \mid N(t)=2 k+1, V(0)= \pm c\} \\
=d x \frac{(2 k+1) !}{(2 c t)^{2 k+1} k !^{2}}\left(c^{2} t^{2}-x^{2}\right)^{k} .
\end{gathered}
$$

Therefore from (2.24) we again obtain formula (2.17). 
Analogously,

$$
\begin{aligned}
& \mathrm{P}\{T(t) \in d x \mid N(t)=2 k+2, V(0)=c\} \\
& =d x \frac{(2 k+2) !}{(2 c t)^{2 k+2} k !(k+1) !}(c t+x)^{k+1}(c t-x)^{k}, \\
& \mathrm{P}\{T(t) \in d x \mid N(t)=2 k+2, V(0)=-c\} \\
& \quad=d x \frac{(2 k+2) !}{(2 c t)^{2 k+2} k !(k+1) !}(c t+x)^{k}(c t-x)^{k+1} .
\end{aligned}
$$

From (2.25a) and (2.25b) we obtain

$$
\begin{aligned}
& \mathrm{P}\{T(t) \in d x \mid N(t)=2 k+2\} \\
& \quad=d x \frac{(2 k+2) !}{2(2 c t)^{2 k+1} k !(k+1) !}\left(c^{2} t^{2}-x^{2}\right)^{k} .
\end{aligned}
$$

The conditional densities above can thus be obtained by means of two different approaches whose degrees of difficulty are equivalent.

\section{REINFORCED ALTERNATING PROCESS}

Here we consider a particle which can move forward and backward with velocity $c$. However we assume that, in general, it can reverse the direction of motion only after two Poisson events. In other words we suppose therefore that the motion has four possible states $s_{j}, j=1, \ldots, 4$, defined as follows:

$$
\left\{\begin{array}{l}
s_{1}=(c, c,-c,-c), \\
s_{2}=(c,-c,-c, c), \\
s_{3}=(-c,-c, c, c), \\
s_{4}=(-c, c, c,-c) .
\end{array}\right.
$$

For example, if the state $s_{1}$ is initially chosen, then the particle moves forward for two consecutive intervals and then backwards for the next two intervals.

At time $t$ the particle lies in the set

$$
\{-c t \leq x \leq c t\}
$$

and can be located at the endpoints $x= \pm c t$ with probability equal to

$$
e^{-\lambda t}+\frac{1}{2} \lambda t e^{-\lambda t}
$$

We denote by $X=X(t)$ the process describing the position of the moving particle at time $t$. If the number of Poisson events occurring in $[0, t]$ is $N(t)=n$, then we have $n_{j}$ steps of type $s_{j}$ with

$$
n+1=n_{1}+n_{2}+n_{3}+n_{4} .
$$

By applying the same considerations on exchangeability as in section 2 we can represent the process $X(t)$, (when $N(t)=n)$ as

$$
X(t)=c t\left\{T_{\left(n_{1}+n_{2}\right)}-\left(1-T_{\left(n_{1}+n_{2}\right)}\right)\right\},
$$

where $T_{\left(n_{1}+n_{2}\right)}$ is the $\left(n_{1}+n_{2}\right)$-th order statistic from the uniform law in $[0,1]$.

From (3.2) we readily have

$$
\begin{aligned}
& \mathrm{P}\left\{X(t)<x \mid N(t)=n, V(0)=s_{j}\right\} \\
& \quad=\mathrm{P}\left\{T_{\left(n_{1}+n_{2}\right)}<\frac{1}{2}\left(1+\frac{x}{c t}\right) \mid N(t)=n, V(0)=s_{j}\right\} .
\end{aligned}
$$


The conditional density of $X(t)$ is therefore

$$
\mathrm{P}\left\{X(t) \in d x \mid N(t)=n, V(0)=s_{j}\right\}=\frac{d x}{2 c t} f_{T_{\left(n_{1}+n_{2}\right)}}\left(\frac{1}{2}\left(1+\frac{x}{c t}\right)\right),
$$

where $f_{T_{\left(n_{1}+n_{2}\right)}}$ is the density of the order statistic (2.22) with $n^{+}$replaced by $n_{1}+n_{2}$. We can write down explicitly the density as follows:

$$
\begin{aligned}
\mathrm{P}\{X(t) & \left.\in d x \mid N(t)=n, V(0)=s_{j}\right\} \\
= & \frac{d x}{2 c t} \frac{n !}{\left(n_{1}+n_{2}-1\right) !\left(n-\left(n_{1}+n_{2}\right)\right) !}\left[\frac{1}{2}\left(1+\frac{x}{c t}\right)\right]^{n_{1}+n_{2}-1} \\
& \times\left[\frac{1}{2}\left(1-\frac{x}{c t}\right)\right]^{n-\left(n_{1}+n_{2}\right)} \\
= & \frac{d x}{(2 c t)^{n}} \frac{n !}{\left(n_{1}+n_{2}-1\right) !\left(n-\left(n_{1}+n_{2}\right)\right) !}(c t+x)^{n_{1}+n_{2}-1}(c t-x)^{n-n_{1}-n_{2}} .
\end{aligned}
$$

By specifying

$$
N(t)=4 k-j, \quad j=0, \ldots, 3, k \geq 1,
$$

and by taking the four possible initial states we have 16 conditional distributions. However only 8 are different and for the convenience of the reader we write them down in the following table:

$$
\begin{aligned}
& \left.\mathrm{P}\left\{X(t) \in d x \mid N(t)=4 k, V(0)=s_{1}\right\}\right\} \\
& \left.\mathrm{P}\left\{X(t) \in d x \mid N(t)=4 k, V(0)=s_{2}\right\}\right\} \\
& =\frac{d x}{(2 c t)^{4 k}} \frac{(4 k) !}{(2 k) !(2 k-1) !}(c t+x)^{2 k}(c t-x)^{2 k-1}, \quad k \geq 1, \\
& \left.\mathrm{P}\left\{X(t) \in d x \mid N(t)=4 k, V(0)=s_{3}\right\}\right\} \\
& \left.\mathrm{P}\left\{X(t) \in d x \mid N(t)=4 k, V(0)=s_{4}\right\}\right\} \\
& =\frac{d x}{(2 c t)^{4 k}} \frac{(4 k) !}{(2 k) !(2 k-1) !}(c t+x)^{2 k-1}(c t-x)^{2 k}, \quad k \geq 1, \\
& \mathrm{P}\left\{X(t) \in d x \mid N(t)=4 k-1, V(0)=s_{j}\right\} \\
& =\frac{d x}{(2 c t)^{4 k-1}} \frac{(4 k-1) !}{(2 k-1) !^{2}}\left(c^{2} t^{2}-x^{2}\right)^{2 k-1}, \quad k \geq 1, j=1,2,3,4, \\
& \left.\mathrm{P}\left\{X(t) \in d x \mid N(t)=4 k-2, V(0)=s_{1}\right\}\right\} \\
& \left.\mathrm{P}\left\{X(t) \in d x \mid N(t)=4 k-2, V(0)=s_{4}\right\}\right\} \\
& =\frac{d x}{(2 c t)^{4 k-2}} \frac{(4 k-2) !}{(2 k-1) !(2 k-2) !}(c t+x)^{2 k-1}(c t-x)^{2 k-2}, \quad k \geq 1, \\
& \left.\mathrm{P}\left\{X(t) \in d x \mid N(t)=4 k-2, V(0)=s_{2}\right\}\right\} \\
& \left.\mathrm{P}\left\{X(t) \in d x \mid N(t)=4 k-2, V(0)=s_{3}\right\}\right\} \\
& =\frac{d x}{(2 c t)^{4 k-2}} \frac{(4 k-2) !}{(2 k-1) !(2 k-2) !}(c t+x)^{2 k-2}(c t-x)^{2 k-1}, \quad k \geq 1,
\end{aligned}
$$




$$
\begin{aligned}
& \mathrm{P}\left\{X(t) \in d x \mid N(t)=4 k-3, V(0)=s_{1}\right\} \\
& =\frac{d x}{(2 c t)^{4 k-3}} \frac{(4 k-3) !}{(2 k-1) !(2 k-3) !}(c t+x)^{2 k-1}(c t-x)^{2 k-3}, \quad k \geq 2, \\
& \mathrm{P}\left\{X(t) \in d x \mid N(t)=4 k-3, V(0)=s_{3}\right\} \\
& =\frac{d x}{(2 c t)^{4 k-3}} \frac{(4 k-3) !}{(2 k-1) !(2 k-3) !}(c t+x)^{2 k-3}(c t-x)^{2 k-1}, \quad k \geq 2, \\
& \left.\mathrm{P}\left\{X(t) \in d x \mid N(t)=4 k-3, V(0)=s_{2}\right\}\right\} \\
& \left.\mathrm{P}\left\{X(t) \in d x \mid N(t)=4 k-3, V(0)=s_{4}\right\}\right\} \\
& =\frac{d x}{(2 c t)^{4 k-3}} \frac{(4 k-3) !}{(2 k-2) !^{2}}\left(c^{2} t^{2}-x^{2}\right)^{2 k-2}, \quad k \geq 1 .
\end{aligned}
$$

The reason that four probabilities (3.7) coincide is that with $N(t)=4 k-1$ there are $4 k$ intervals and thus all states $s_{j}$ are occupied exactly $k$ times. If $N(t) \neq 4 k-1$, the states $s_{j}$ are occupied a different number of times because the last cycle of motion is, in this case, incomplete. Another feature emerging from the formulas above is that some distributions are asymmetric (10 out of 16 ) because the number of steps forward and backward, in general, do not coincide. By summing up the above distributions we get the four important conditional densities of the following table:

$$
\begin{gathered}
\mathrm{P}\{X(t) \in d x \mid N(t)=4 k\}=\mathrm{P}\{X(t) \in d x \mid N(t)=4 k-1\} \\
=\frac{d x}{(2 c t)^{4 k-1}} \frac{(4 k-1) !}{(2 k-1) !^{2}}\left(c^{2} t^{2}-x^{2}\right)^{2 k-1}, \\
k \geq 1, \quad|x|<c t, \\
\mathrm{P}\{X(t) \in d x \mid N(t)=4 k-2\} \\
=\frac{d x}{2(2 c t)^{4 k-3}} \frac{(4 k-2) !}{(2 k-1) !(2 k-2) !}\left(c^{2} t^{2}-x^{2}\right)^{2 k-2}, \\
k \geq 1, \quad|x|<c t, \\
\mathrm{P}\{X(t) \in d x \mid N(t)=4 k-3\} \quad x^{2 k}, x^{2 k-3} \\
=\frac{d x}{(2 c t)^{4 k-3}} \frac{(4 k-3) !}{(2 k-1) !}\left\{\frac{\left(c^{2} t^{2}-x^{2}\right)^{2 k-2}}{2(2 k-2) !}+\frac{c^{2} t^{2}\left(c^{2} t^{2}-x^{2}\right)^{2 k-3}}{(2 k-3) !}\right\}, \\
k \geq 1, \quad|x|<c t .
\end{gathered}
$$

The last distribution necessitates some manipulations in order to be obtained. It is clear that by eliminating the condition $V(0)=s_{j}$, we get symmetric densities. The reader can be surprised that the probabilities (3.10a) coincide. This coincidence has been noted also for the telegraph process (see formula (2.19)) as well as in the case of motion with two stops (see 4], formula (3.4)). 
Remark 3.1. The distributions (3.10) integrate to one as can be checked by taking into account the following formulas:

$$
\begin{aligned}
\int_{-c t}^{c t}\left(c^{2} t^{2}-x^{2}\right)^{2 k-2} d x & =(c t)^{4 k-3} \int_{0}^{1}(1-y)^{2 k-2} y^{1 / 2-1} d y \\
& =(c t)^{4 k-3} \frac{\Gamma(2 k-1)}{2} \frac{\Gamma(1 / 2)(4 k-1)}{\Gamma(2 k+1 / 2)} \\
& =(c t)^{4 k-3} \frac{\Gamma(2 k-1)}{2} \frac{\Gamma(2 k)(4 k-1)}{\Gamma(4 k) 2^{1-4 k}} .
\end{aligned}
$$

Analogously we have

$$
\int_{-c t}^{c t}\left(c^{2} t^{2}-x^{2}\right)^{2 k-3} d x=(c t)^{4 k-5} \frac{\Gamma^{2}(2 k-2)}{2^{5-4 k} \Gamma(4 k-4)} .
$$

Theorem 3.1. The absolutely continuous part of the distribution of the process $X=X(t)$ is

$$
\begin{aligned}
& \mathrm{P}\{X(t) \in d x\} \\
& =e^{-\lambda t} d x \sum_{k=1}^{\infty}\left\{\frac{\lambda t}{4 k}+1+\frac{1}{\lambda} \frac{\partial}{\partial t}+\frac{1}{\lambda^{2}} \frac{\partial^{2}}{\partial t^{2}}\right\}\left(\frac{\lambda}{2 c}\right)^{4 k-1} \frac{\left(c^{2} t^{2}-x^{2}\right)^{2 k-1}}{(2 k-1) !^{2}} \\
& =e^{-\lambda t} d x\left[\sum_{k=1}^{\infty} \frac{\lambda t}{4 k}\left(\frac{\lambda}{2 c}\right)^{4 k-1} \frac{\left(c^{2} t^{2}-x^{2}\right)^{2 k-1}}{(2 k-1) !^{2}}\right. \\
& +\sum_{k=1}^{\infty}\left(\frac{\lambda}{2 c}\right)^{4 k-1} \frac{\left(c^{2} t^{2}-x^{2}\right)^{2 k-1}}{(2 k-1) !^{2}} \\
& +\frac{\lambda t}{2} \sum_{k=1}^{\infty}\left(\frac{\lambda}{2 c}\right)^{4 k-3} \frac{\left(c^{2} t^{2}-x^{2}\right)^{2 k-2}}{(2 k-1) !(2 k-2) !} \\
& +\frac{1}{2} \sum_{k=1}^{\infty}\left(\frac{\lambda}{2 c}\right)^{4 k-3} \frac{\left(c^{2} t^{2}-x^{2}\right)^{2 k-2}}{(2 k-1) !(2 k-2) !} \\
& \left.+c^{2} t^{2} \sum_{k=2}^{\infty}\left(\frac{\lambda}{2 c}\right)^{4 k-3} \frac{\left(c^{2} t^{2}-x^{2}\right)^{2 k-3}}{(2 k-3) !(2 k-1) !}\right]
\end{aligned}
$$

Furthermore,

$$
\int_{-c t}^{c t} \mathrm{P}\{X(t) \in d x\}=1-e^{-\lambda t}-\frac{\lambda t}{2} e^{-\lambda t} .
$$

Proof. It suffices to sum up the conditional densities (3.10) multiplied by

$$
\mathrm{P}\{N(t)=4 k-j\}=e^{-\lambda t} \frac{(\lambda t)^{4 k-j}}{(4 k-j) !}, \quad j=0, \ldots, 3 .
$$

In order to check the boundary condition (3.14), we must take into account, in addition to (3.11) and (3.12), that

$$
\int_{-c t}^{c t}\left(c^{2} t^{2}-x^{2}\right)^{2 k-1} d x=(c t)^{4 k-1} \frac{(2 k-1) !^{2} 2^{4 k-1}}{(4 k-1) !} .
$$


Therefore it is clear:

$$
\begin{aligned}
& \int_{-c t}^{c t} \mathrm{P}\{X(t) \in d x\} \\
&=e^{-\lambda t}\left[\sum_{k=1}^{\infty} \frac{(\lambda t)^{4 k}}{(4 k) !}+\sum_{k=1}^{\infty} \frac{(\lambda t)^{4 k-1}}{(4 k-1) !}+\sum_{k=1}^{\infty} \frac{(\lambda t)^{4 k-2}}{(4 k-2) !}\right. \\
&\left.\quad+\sum_{k=1}^{\infty} \frac{(\lambda t)^{4 k-3}}{(4 k-2) !}+\frac{1}{2} \sum_{k=2}^{\infty} \frac{(\lambda t)^{4 k-3}}{(2 k-1)(4 k-4) !}\right] \\
&=e^{-\lambda t}\left[\sum_{k=1}^{\infty} \frac{(\lambda t)^{4 k}}{(4 k) !}+\sum_{k=1}^{\infty} \frac{(\lambda t)^{4 k-1}}{(4 k-1) !}+\sum_{k=1}^{\infty} \frac{(\lambda t)^{4 k-2}}{(4 k-2) !}\right. \\
&\left.\quad+\sum_{k=1}^{\infty} \frac{(\lambda t)^{4 k-3}}{(4 k-2)(4 k-4) !}\left\{1+\frac{1}{4 k-3}\right\}\right]-\frac{\lambda t}{2} e^{-\lambda t} \\
&=e^{-\lambda t}\left[\sum_{k=1}^{\infty} \frac{(\lambda t)^{4 k}}{(4 k) !}+\sum_{k=1}^{\infty} \frac{(\lambda t)^{4 k-1}}{(4 k-1) !}+\sum_{k=1}^{\infty} \frac{(\lambda t)^{4 k-2}}{(4 k-2) !}+\sum_{k=1}^{\infty} \frac{(\lambda t)^{4 k-3}}{(4 k-3) !}\right]-\frac{\lambda t}{2} e^{-\lambda t} \\
&=1-e^{-\lambda t}-\frac{\lambda t}{2} e^{-\lambda t} .
\end{aligned}
$$

We point out that the endpoint $x=c t$ is reached with probability equal to

$$
\frac{1}{4} e^{-\lambda t}+\frac{\lambda t}{4} e^{-\lambda t}
$$

if the initial state is $s_{1}$. However if the initial state is $s_{2}$, the endpoint $x=c t$ can be reached only if no Poisson event occurs, that is, with probability $\frac{1}{4} e^{-\lambda t}$.

Remark 3.2. The distribution (3.13) is a solution to the fourth-order equation

$$
\left(\frac{\partial^{2}}{\partial t^{2}}+2 \lambda \frac{\partial}{\partial t}-c^{2} \frac{\partial^{2}}{\partial x^{2}}\right)\left(\frac{\partial^{2}}{\partial t^{2}}+2 \lambda \frac{\partial}{\partial t}-c^{2} \frac{\partial^{2}}{\partial x^{2}}+2 \lambda^{2}\right) p=0
$$

which, after the usual exponential transformation $p=e^{-\lambda t} w$, takes the form

$$
\left(\frac{\partial^{2}}{\partial t^{2}}-c^{2} \frac{\partial^{2}}{\partial x^{2}}-\lambda^{2}\right)\left(\frac{\partial^{2}}{\partial t^{2}}-c^{2} \frac{\partial^{2}}{\partial x^{2}}+\lambda^{2}\right) w=0
$$

By means of the transformation $s=\sqrt{c^{2} t^{2}-x^{2}}$ equation (3.17) can be converted into the biquadratic Bessel equation

$$
\left(\frac{\partial^{2}}{\partial s^{2}}+\frac{1}{s} \frac{\partial}{\partial s}-\frac{\lambda^{2}}{c^{2}}\right)\left(\frac{\partial^{2}}{\partial s^{2}}+\frac{1}{s} \frac{\partial}{\partial s}+\frac{\lambda^{2}}{c^{2}}\right) w=0 .
$$

\section{Cyclic planar motion With three Directions}

A planar motion with three directions (represented by the vectors $d_{1}=(1,0), d_{2}=$ $\left.(-1 / 2, \sqrt{3} / 2), d_{3}=(-1 / 2,-\sqrt{3} / 2)\right)$ taken cyclically at Poisson paced times is described and analyzed in [5]. In [3] the general planar motion with three directions is examined by using order statistics. 
The following decomposition of the continuous part of the distribution holds true:

$$
\begin{aligned}
& \mathrm{P}\{X(t) \in d x, Y(t) \in d y\} \\
& =\sum_{k=2}^{\infty} \mathrm{P}\{N(t)=k\} \\
& \quad \times \sum_{n_{0}=1}^{k-1} \sum_{n_{1}=1}^{k-n_{0}} \mathrm{P}\left\{N_{0}=n_{0}, N_{1}=n_{1}, N_{2}=k+1-n_{0}-n_{1} \mid N(t)=k\right\} \\
& \quad \times \mathrm{P}\{X(t) \in d x, Y(t) \in d y \mid \\
& \left.\quad N(t)=k, N_{0}=n_{0}, N_{1}=n_{1}, N_{2}=k+1-n_{0}-n_{1}\right\},
\end{aligned}
$$

where $\left(N_{0}, N_{1}, N_{2}\right)$ is the number of times the directions $d_{0}, d_{1}$, and $d_{2}$ are taken (with $\left.N(t)+1=N_{0}+N_{1}+N_{2}\right)$.

For the cyclic case, formula (4.1) considerably simplifies because the random content of motion reduces to the time spent moving in each direction, which reflects into the last factor of (4.1).

By means of order statistics it can be seen that

$$
\begin{aligned}
\mathrm{P}\{X(t) & \left.\in d x, Y(t) \in d y \mid N(t)=k, N_{0}=n_{0}, N_{1}=n_{1}, N_{2}=k+1-n_{0}-n_{1}\right\} \\
= & d x d y \frac{2 \cdot 3}{\sqrt{3}} \\
& \times \frac{k !}{(3 c t)^{k}} \frac{(c t+2 x)^{n_{0}-1}(c t-x+y \sqrt{3})^{n_{1}-1}(c t-x-y \sqrt{3})^{k-n_{0}-n_{1}}}{\left(n_{0}-1\right) !\left(n_{1}-1\right) !\left(k-n_{0}-n_{1}\right) !}
\end{aligned}
$$

for $(x, y) \in \operatorname{int}\left\{T_{c t}\right\}$, where

$$
T_{c t}=\left\{x, y:-\frac{c t}{2} \leq x \leq \frac{c t}{2},-\frac{c t-x}{\sqrt{3}} \leq y \leq \frac{c t-x}{\sqrt{3}}\right\},
$$

$n_{0} \geq 1, n_{1} \geq 1, k \geq 2$. The proof of this result is in [3].

In the special case of cyclic motion, formula (4.2) specializes in the cases reported in the next theorem.

Theorem 4.1. For $(x, y) \in \operatorname{int}\left\{T_{c t}\right\}$,

$$
\begin{aligned}
& \mathrm{P}\{X(t) \in d x, Y(t) \in d y \mid N(t)=3 n-1\} \\
& =d x d y \frac{(3 n-1) !}{(3 c t)^{3 n-1}} \frac{2 \cdot 3}{\sqrt{3}} \frac{\left[(c t+2 x)\left((c t-x)^{2}-3 y^{2}\right)\right]^{n-1}}{[(n-1) !]^{3}}, \quad n \geq 1, \\
& \mathrm{P}\left\{X(t) \in d x, Y(t) \in d y \mid N(t)=3 n-2, D(0)=d_{0}\right\} \\
& =d x d y \frac{(3 n-2) !}{(3 c t)^{3 n-2}} \frac{2 \cdot 3}{\sqrt{3}} \frac{(c t+2 x)^{n-1}(c t-x+y \sqrt{3})^{n-1}(c t-x-y \sqrt{3})^{n-2}}{[(n-1) !]^{2}(n-2) !} \\
& \mathrm{P}\left\{X(t) \in d x, Y(t) \in d y \mid N(t)=3 n-2, D(0)=d_{1}\right\} \\
& =d x d y \frac{(3 n-2) !}{(3 c t)^{3 n-2}} \frac{2 \cdot 3}{\sqrt{3}} \frac{(c t+2 x)^{n-2}(c t-x+y \sqrt{3})^{n-1}(c t-x-y \sqrt{3})^{n-1}}{[(n-1) !]^{2}(n-2) !} \\
& n \geq 2,
\end{aligned}
$$




$$
\begin{aligned}
& \mathrm{P}\left\{X(t) \in d x, Y(t) \in d y \mid N(t)=3 n-2, D(0)=d_{2}\right\} \\
& =d x d y \frac{(3 n-2) !}{(3 c t)^{3 n-2}} \frac{2 \cdot 3}{\sqrt{3}} \frac{(c t+2 x)^{n-1}(c t-x+y \sqrt{3})^{n-2}(c t-x-y \sqrt{3})^{n-1}}{[(n-1) !]^{2}(n-2) !}, \\
& n \geq 2, \\
& \mathrm{P}\left\{X(t) \in d x, Y(t) \in d y \mid N(t)=3 n-3, D(0)=d_{0}\right\} \\
& \quad=d x d y \frac{(3 n-3) !}{(3 c t)^{3 n-3}} \frac{2 \cdot 3}{\sqrt{3}} \frac{(c t+2 x)^{n-1}(c t-x+y \sqrt{3})^{n-2}(c t-x-y \sqrt{3})^{n-2}}{[(n-2) !]^{2}(n-1) !} \\
& \mathrm{P}\left\{X(t) \in d x, Y(t) \in d y \mid N(t)=3 n-3, D(0)=d_{1}\right\} \\
& \quad=d x d y \frac{(3 n-3) !}{(3 c t)^{3 n-3}} \frac{2 \cdot 3}{\sqrt{3}} \frac{(c t+2 x)^{n-2}(c t-x+y \sqrt{3})^{n-1}(c t-x-y \sqrt{3})^{n-2}}{[(n-2) !]^{2}(n-1) !} \\
& \mathrm{P}\left\{X(t) \in d x, Y(t) \in d y \mid N(t)=3 n-3, D(0)=d_{2}\right\} \\
& \quad=d x d y \frac{(3 n-3) !}{(3 c t)^{3 n-3}} \frac{2 \cdot 3}{\sqrt{3}} \frac{(c t+2 x)^{n-2}(c t-x+y \sqrt{3})^{n-2}(c t-x-y \sqrt{3})^{n-1}}{[(n-2) !]^{2}(n-1) !} \\
& n \geq 2 .
\end{aligned}
$$

Proof. The case of (4.3a) corresponds to the complete cycle where each direction is taken $n$ times and thus the initial direction $D(0)$ plays no role. Formulas (4.3b), (4.3c) and $(4.3 \mathrm{~d})$ concern the case where the incomplete cycles differ because two directions are taken $n$ times and one $n-1$ times according to the initial direction. The other three formulas refer to the case where two directions are taken $n-1$ times and one $n$ times.

The distributions follow from (4.2) by specializing the numbers $n_{0}, n_{1}$, and $n_{2}$ accordingly.

From Theorem 4.1 we easily infer the conditional distributions, independent from the initial directions.

Theorem 4.2. For $(x, y) \in \operatorname{int}\left\{T_{c t}\right\}$,

$$
\begin{aligned}
& \mathrm{P}\{X(t) \in d x, Y(t) \in d y \mid N(t)=3 n-1\} \\
& \quad=d x d y \frac{(3 n-1) !}{(3 c t)^{3 n-1}} \frac{2 \cdot 3}{\sqrt{3}} \frac{\left[(c t+2 x)\left((c t-x)^{2}-3 y^{2}\right)\right]^{n-1}}{[(n-1) !]^{3}}, \quad n \geq 1, \\
& \mathrm{P}\{X(t) \in d x, Y(t) \in d y \mid N(t)=3 n-2\} \\
& \quad=d x d y \frac{(3 n-2) !}{(3 c t)^{3 n-2}} \frac{2 \cdot 3}{\sqrt{3}} \frac{\left(c^{2} t^{2}-x^{2}-y^{2}\right)\left[(c t+2 x)\left((c t-x)^{2}-3 y^{2}\right)\right]^{n-2}}{[(n-1) !]^{2}(n-2) !}, \\
& \mathrm{P}\{X(t) \in d x, Y(t) \in d y \mid N(t)=3 n-3\} \\
& \quad=d x d y \frac{(3 n-3) !}{(3 c t)^{3 n-3}} \frac{2 \cdot 3}{\sqrt{3}} c t \frac{\left[(c t+2 x)\left((c t-x)^{2}-3 y^{2}\right)\right]^{n-2}}{[(n-2) !]^{2}(n-1) !}, \quad n \geq 2 .
\end{aligned}
$$

Proof. It is sufficient to consider that each initial direction is taken with probability $\frac{1}{3}$ and then use the formulas of Theorem 4.1. 
Remark 4.1. We now examine some special cases of the conditional distributions appearing in Theorem 4.2:

$$
\begin{gathered}
\mathrm{P}\{X(t) \in d x, Y(t) \in d y \mid N(t)=2\}=\frac{4 d x d y}{3 \sqrt{3} c^{2} t^{2}}, \quad(x, y) \in T_{c t}, \\
\mathrm{P}\{X(t) \in d x, Y(t) \in d y \mid N(t)=3\}=\frac{4 d x d y}{3 \sqrt{3} c^{2} t^{2}}, \quad(x, y) \in T_{c t}, \\
\mathrm{P}\{X(t) \in d x, Y(t) \in d y \mid N(t)=4\}=\frac{2 \cdot 3}{\sqrt{3}} \frac{4 !}{(3 c t)^{4}}\left(c^{2} t^{2}-x^{2}-y^{2}\right) d x d y, \\
=\frac{2}{\sqrt{3}} \frac{4 !}{(3 c t)^{4}}\left[(c t-x)^{2}-3 y^{2}+2(c t-x)(c t+2 x)\right] d x d y, \quad(x, y) \in T_{c t}, \\
\mathrm{P}\{X(t) \in d x, Y(t) \in d y \mid N(t)=5\} \\
=\frac{2 \cdot 3}{\sqrt{3}} \frac{5 !}{(3 c t)^{5}}\left[(c t+2 x)\left((c t-x)^{2}-3 y^{2}\right)\right] .
\end{gathered}
$$

The first two distributions are uniform in the triangle $T_{c t}$. Perhaps it is worthwhile to note that when $N(t)=3$ and the initial direction $D(0)$ is fixed, the conditional distribution is not uniform because the direction $D(0)$ gives an initial advantage to some part of the set $T_{c t}$ which is compensated when taking the average.

When $N(t)=4$ (incomplete cycle with one direction taken one time less than the others) we see that the distribution does not vanish on the border $\partial T_{c t}$.

For $N(t)=5$ the density is tied down to zero all along the border because the sample paths coil up so that the particle hardly leaves the starting point.

The distribution (4.5d) coincides with

$$
\mathrm{P}\{X(t) \in d x, Y(t) \in d y \mid N(t)=6\}
$$

as can be easily checked from (4.4c) for $n=3$. The same coincidence is repeated for the conditional probabilities where $N(t)=3 n-1$ and $N(t)=3 n$.

Theorem 4.3. We have the following probabilities for $(x, y) \in \operatorname{int}\left\{T_{c t}\right\}$ :

$$
\begin{aligned}
& \sum_{n=1}^{\infty} \mathrm{P}\{X(t) \in d x, Y(t) \in d y, N(t)=3 n-1\} \\
& =\mathrm{P}\{(X(t) \in d x, Y(t) \in d y) \cap \text { (the cycle is complete })\} \\
& =e^{-\lambda t} \frac{2 \lambda^{2}}{3 \sqrt{3} c^{2}} I_{0,3}\left(\frac{\lambda}{c} \sqrt[3]{(c t+2 x)\left((c t-x)^{2}-3 y^{2}\right)}\right) d x d y, \\
& \sum_{n=2}^{\infty} \mathrm{P}\{X(t) \in d x, Y(t) \in d y, N(t)=3 n-2\} \\
& =e^{-\lambda t} \frac{2 \lambda}{3 \sqrt{3} c^{2}} \frac{\partial}{\partial t} I_{0,3}\left(\frac{\lambda}{c} \sqrt[3]{(c t+2 x)\left((c t-x)^{2}-3 y^{2}\right)}\right) d x d y \\
& \sum_{n=2}^{\infty} \mathrm{P}\{X(t) \in d x, Y(t) \in d y, N(t)=3 n-3\} \\
& =e^{-\lambda t} \frac{6 c t}{\sqrt{3}}\left(\frac{\lambda}{3 c}\right)^{2} \frac{I_{0,3}^{1,0}\left(\frac{\lambda}{c} \sqrt[3]{(c t+2 x)\left((c t-x)^{2}-3 y^{2}\right)}\right)}{\sqrt[3]{(c t+2 x)\left((c t-x)^{2}-3 y^{2}\right)}} d x d y
\end{aligned}
$$


where

$$
I_{0,3}(x)=\sum_{k=0}^{\infty}\left(\frac{x}{3}\right)^{3 k} \frac{1}{(k !)^{3}} \quad \text { and } \quad I_{0,3}^{1,0}(x)=\sum_{k=0}^{\infty}\left(\frac{x}{3}\right)^{3 k+1} \frac{1}{(k !)^{2}(k+1) !}
$$

are Bessel functions of order three.

Proof. Since the derivation of (4.6a) appears also in [3, we give some details of the other two distributions.

From the formulas of Theorem 4.1 we have

$$
\begin{aligned}
& \sum_{n=2}^{\infty} \mathrm{P}\{X(t) \in d x, Y(t) \in d y, N(t)=3 n-2\} \\
& =d x d y \frac{e^{-\lambda t}}{3} \\
& \times \sum_{n=2}^{\infty} \frac{(\lambda t)^{3 n-2}}{(3 n-2) !}\left\{\frac{2 \cdot 3}{\sqrt{3}} \frac{(3 n-2) !}{(3 c t)^{3 n-2}} \frac{1}{[(n-1) !]^{2}(n-2) !}\right. \\
& \times\left[(c t+2 x)^{n-1}(c t-x+y \sqrt{3})^{n-1}(c t-x-y \sqrt{3})^{n-2}\right. \\
& +(c t+2 x)^{n-2}(c t-x+y \sqrt{3})^{n-1}(c t-x-y \sqrt{3})^{n-1} \\
& \left.\left.+(c t+2 x)^{n-1}(c t-x+y \sqrt{3})^{n-2}(c t-x-y \sqrt{3})^{n-1}\right]\right\} \\
& =d x d y \frac{e^{-\lambda t}}{\sqrt{3}} 2 \sum_{r=1}^{\infty}\left(\frac{\lambda}{3 c}\right)^{3 r+1} \frac{1}{(r !)^{2}(r-1) !} \\
& \times\left[(c t+2 x)^{r}(c t-x+y \sqrt{3})^{r}(c t-x-y \sqrt{3})^{r-1}\right. \\
& +(c t+2 x)^{r-1}(c t-x+y \sqrt{3})^{r}(c t-x-y \sqrt{3})^{r} \\
& \left.+(c t+2 x)^{r}(c t-x+y \sqrt{3})^{r-1}(c t-x-y \sqrt{3})^{r}\right] \\
& =\frac{d x d y 2 e^{-\lambda t}}{c \sqrt{3}} \frac{\lambda}{3 c} \sum_{r=0}^{\infty}\left(\frac{\lambda}{3 c}\right)^{3 r} \frac{1}{(r !)^{3}} \frac{\partial}{\partial t}(c t+2 x)^{r}(c t-x+y \sqrt{3})^{r}(c t-x-y \sqrt{3})^{r} \\
& =\frac{d x d y 2 \lambda e^{-\lambda t}}{3 \sqrt{3} c^{2}} \frac{\partial}{\partial t} I_{0,3}\left(\frac{\lambda}{c} \sqrt[3]{(c t+2 x)\left((c t-x)^{2}-3 y^{2}\right)}\right) .
\end{aligned}
$$

As far as the last formula of this theorem is concerned we have

$$
\begin{aligned}
\sum_{n=2}^{\infty} \mathrm{P}\{X(t) \in x, Y(t) \in d y, N(t) & =3 n-3\} \\
=\frac{d x d y}{3} e^{-\lambda t} \sum_{n=2}^{\infty} \frac{(\lambda t)^{3 n-3}}{(3 n-3) !}\{ & \frac{2 \cdot 3}{\sqrt{3}} \frac{(3 n-3) !}{(3 c t)^{3 n-3}} \frac{1}{[(n-2) !]^{2}(n-1) !} \\
\times & {\left[(c t+2 x)^{n-1}(c t-x+y \sqrt{3})^{n-2}(c t-x-y \sqrt{3})^{n-2}\right.} \\
& +(c t+2 x)^{n-2}(c t-x+y \sqrt{3})^{n-1}(c t-x-y \sqrt{3})^{n-2} \\
& \left.\left.+(c t+2 x)^{n-2}(c t-x+y \sqrt{3})^{n-2}(c t-x-y \sqrt{3})^{n-1}\right]\right\}
\end{aligned}
$$




$$
\begin{aligned}
\begin{aligned}
&=d x d y \frac{2}{\sqrt{3}}\left(\frac{\lambda}{3 c}\right)^{3} e^{-\lambda t} \sum_{r=0}^{\infty}(\left(\frac{\lambda}{3 c}\right)^{3 r} \frac{1}{(r !)^{2}(r+1) !} \\
& \times {\left[(c t+2 x)^{r+1}(c t-x+y \sqrt{3})^{r}(c t-x-y \sqrt{3})^{r}\right.} \\
&+(c t+2 x)^{r}(c t-x+y \sqrt{3})^{r+1}(c t-x-y \sqrt{3})^{r} \\
&\left.+(c t+2 x)^{r}(c t-x+y \sqrt{3})^{r}(c t-x-y \sqrt{3})^{r+1}\right] \\
&=d x d y \frac{2}{\sqrt{3}}\left(\frac{\lambda}{3 c}\right)^{3} e^{-\lambda t} \sum_{k=0}^{\infty}\left(\frac{\lambda}{3 c}\right)^{3 k} \frac{3 c t}{(k !)^{2}(k+1) !}\left[(c t+2 x)\left((c t-x)^{2}-3 y^{2}\right)\right]^{k} \\
&=d x d y \frac{6 c t}{\sqrt{3}}\left(\frac{\lambda}{3 c}\right)^{2} e^{-\lambda t} \frac{I_{0,3}^{1,0}(}{\left(\frac{\lambda}{c} \sqrt[3]{(c t+2 x)\left((c t-x)^{2}-3 y^{2}\right)}\right)} \\
& \sqrt[3]{(c t+2 x)\left((c t-x)^{2}-3 y^{2}\right)}
\end{aligned}
\end{aligned}
$$

Remark 4.2. Since all the conditional distributions integrate to 1 it can be easily seen that

$$
\begin{aligned}
& \iint_{T_{c t}} \sum_{j=2}^{3} \sum_{n=2}^{\infty} \mathrm{P}\{X(t) \in d x, Y(t) \in d y, N(t)=3 n-j\} \\
& \quad+\iint_{T_{c t}} \sum_{n=1}^{\infty} \mathrm{P}\{X(t) \in d x, Y(t) \in d y, N(t)=3 n-1\} \\
& =\sum_{j=2}^{3} \sum_{n=2}^{\infty} \mathrm{P}\{N(t)=3 n-j\}+\sum_{n=1}^{\infty} \mathrm{P}\{N(t)=3 n-1\} \\
& =\mathrm{P}\left\{\bigcup_{k=2}^{\infty}(N(t)=k)\right\}=1-e^{-\lambda t}-\lambda t e^{-\lambda t} .
\end{aligned}
$$

The missing probability pertains to the singular component of the distribution and is concentrated on $\partial T_{c t}$.

We also note that while the two terms (4.6a) and (4.6b) coincide with the first and second term of formula (3.1) of [5], the third term differs. Although (4.6c) can be expressed in terms of the Bessel function $I_{0,3}$ it does not coincide with the second derivative there. The first two formulas were obtained in [5] by means of an analytical approach which proved to be successful only partially.

\section{BIBLIOGRAPHY}

1. S. K. Foong and S. Kanno, Properties of the telegrapher's random process with or without a trap, Stochastic Processes and their Applications 53 (1994), 147-173. MR.1290711 (95g:60089)

2. S. Goldstein, On diffusion by discontinuous movements and telegraph equation, Quart. J. Mech. Appl. Math. 4 (1951), 129-156. MR0047963 (13,960b)

3. S. Leorato and E. Orsingher, Bose-Einstein-type statistics, order statistics and planar random motions with three directions, Advances in Applied Probability 36 (2004), no. 3, 937-970. MR2079922 (2005g:60171)

4. S. Leorato, E. Orsingher, and M. Scavino, An alternating motion with stops and the related planar, cyclic motion with four directions, Advances in Applied Probability 35 (2003), no. 4, 1153-1168. MR2014274 (2004g:60132)

5. E. Orsingher, Bessel functions of third order and the distribution of cyclic planar motions with three directions, Stochastics and Stochastics Reports 74 (2002), 617-631. MR1943582 (2003j:60144) 
6. I. V. Samoilenko, Markovian random evolutions in $R^{n}$, Random Operators and Stochastic Equations 9 (2001), 139-160. MR.1832161 (2002e:60116)

7. A. F. Turbin and D. N. Plotkin, Bessel equations and functions of higher order, Asymptotic Methods in Problems of Random Evolutions, Institute of Mathematics, Kiev, 1991, pp. $112-121$. MR:1190488 (94e:35045)

Dipartimento di Scienze Statistiche, University of Padua, via Cesare Battisti 241, 35121, PADUA, ITALY

Dipartimento di Statistica, Probabilità e Statistiche Applicate, University of Rome "La SAPIEnZA", P. Le Aldo Moro 5, 00185, Rome, Italy

E-mail address: enzo.orsingher@uniroma1.it

Department of Probability Theory and Mathematical Statistics, Kyiv National Taras Shevchenko University, Volodymyrska 64, 01033, Kyiv, Ukraine

Received $17 / \mathrm{JUN} / 2003$

Originally published in English 